\title{
Cachexia and Dietetic Interventions in Patients With Esophagogastric Cancer: A Multicenter Cohort Study
}

\author{
Willemieke P.M. Dijksterhuis, MD 1,2; Anouk E.J. Latenstein, MD³; Jessy Joy van Kleef, MSc ${ }^{1}$; Rob H.A. Verhoeven, PhD²; \\ Jeanne H.M. de Vries, PhD"; Marije Slingerland, MD, PhD ${ }^{5}$; Elles Steenhagen, BSc ${ }^{6}$; Joos Heisterkamp, MD, PhD7; \\ Liesbeth M. Timmermans, PhD ${ }^{8,9}$; Marian A.E. de van der Schueren, $\mathrm{PhD}^{10}$; Martijn G.H. van Oijen, PhD ${ }^{1,2}$; \\ Sandra Beijer, $\mathrm{PhD}^{2, \star}$; and Hanneke W.M. van Laarhoven, MD, $\mathrm{PhD}^{1, *}$
}

\section{ABSTRACT}

Background: Cachexia is common in patients with esophagogastric cancer and is associated with increased mortality. Nutritional screening and dietetic interventions can be helpful in preventing evolvement of cachexia. Our aim was to study the real-world prevalence and prognostic value of pretreatment cachexia on overall survival (OS) using patient-reported weight loss, and to explore dietetic interventions in esophagogastric cancer. Materials and Methods: Patients with esophagogastric cancer (2015-2018), regardless of disease stage, who participated in the Prospective Observational Cohort Study of Esophageal-Gastric Cancer Patients (POCOP) and completed patient-reported outcome measures were included. Data on weight loss and dietetic interventions were retrieved from questionnaires before start of treatment (baseline) and 3 months thereafter. Additional patient data were obtained from the Netherlands Cancer Registry. Cachexia was defined as self-reported $>5 \%$ half-year body weight loss at baseline or $>2 \%$ in patients with a body mass index (BMI) $<20 \mathrm{~kg} / \mathrm{m}^{2}$ according to the Fearon criteria. The association between cachexia and OS was analyzed using multivariable Cox proportional hazard analyses adjusted for sex, age, performance status, comorbidities, primary tumor location, disease stage, histology, and treatment strategy. Results: Of 406 included patients, $48 \%$ had pretreatment cachexia, of whom $65 \%$ were referred for dietetic consultation at baseline. The proportion of patients with cachexia was the highest among those who received palliative chemotherapy (59\%) or best supportive care (67\%). Cachexia was associated with decreased OS (hazard ratio, 1.52; $95 \% \mathrm{Cl}, 1.11-2.09$ ). Median weight loss after 3-month follow-up was lower in patients with cachexia who were referred to a dietician at baseline compared with those who were not $(0 \%$ vs $2 \% ; P=.047)$. Conclusions: Nearly half of patients with esophagogastric cancer have pretreatment cachexia. Dietetic consultation at baseline was not reported in more than one-third of the patients with cachexia. Because cachexia was independently associated with decreased survival, improving nutritional screening and referral for dietetic consultation are warranted to prevent further deterioration of malnutrition and mortality.

J Natl Compr Canc Netw 2021;19(2):144-152 doi: $10.6004 /$ jnccn.2020.7615

See JNCCN.org for supplemental online content.

\section{Background}

Malnutrition is defined as a state resulting from lack of intake or uptake of nutrition that leads to altered body composition and body cell mass, and is associated with diminished physical and mental function and impaired clinical outcome. ${ }^{1,2}$ It is reported in $48 \%$ to $85 \%$ of patients with esophagogastric cancer, ${ }^{3-6}$ and is caused by reduced dietary intake as a result of dysphagia and odynophagia caused by tumor obstruction; treatment-related toxicity, such as mucositis, nausea, or taste changes, possibly aggravated by tumor-induced anorexia; and psychologic distress. ${ }^{3,7-11}$ Reduced dietary intake, together with decreased physical activity and tumor-induced catabolism, are causes of cancer-related cachexia. ${ }^{12}$ Cachexia is a multifactorial syndrome characterized by involuntary weight loss due to ongoing loss of skeletal muscle mass, that can only partly be reversed by conventional nutritional support. ${ }^{13,14}$ It is associated with poorer compliance to chemotherapy, increased treatment toxicity, lower quality of life, and even decreased survival in patients with cancer. ${ }^{12-22}$ Patients with cancer and major ( $>5 \%$ of half-year body weight) or minor weight loss $(>2 \%)$ but a low body mass index (BMI) are assumed to have cachexia. ${ }^{13}$

1'Department of Medical Oncology, Amsterdam UMC, University of Amsterdam, Cancer Center Amsterdam, Amsterdam; ${ }^{2}$ Department of Research and Development, Netherlands Comprehensive Cancer Organisation, Amsterdam; ${ }^{3}$ Department of Surgery, Amsterdam UMC, University of Amsterdam, Cancer Center Amsterdam, Amsterdam; ${ }^{4}$ Division of Human Nutrition and Health, Wageningen University, Wageningen; ${ }^{5}$ Department of Medical Oncology, Leiden University Medical Center, Leiden; ${ }^{6}$ Department of Dietetics, University Medical Center Utrecht, Utrecht; ${ }^{7}$ Department of Surgery, Elisabeth-

TweeSteden Hospital, Tilburg; ${ }^{8}$ Stichting voor Patiënten met Kanker aan het Spijsverteringskanaal, Utrecht; ${ }^{9}$ Department of Primary and Community Care, Radboud University Medical Centre, Nijmegen; and ${ }^{10}$ Department of Nutrition and Health, HAN University of Applied Sciences, Nijmegen, the Netherlands.

${ }^{*}$ Contributed equally as senior authors.

See page 235 for related commentary. 
According to an international consensus statement included in the European Society for Clinical Nutrition and Metabolism (ESPEN) nutrition guideline, all patients with cancer should be screened for the risk or presence of malnutrition. ${ }^{2}$ Patients can be identified as at high risk of malnutrition using validated screening tools, such as the Short Nutritional Assessment Questionnaire (SNAQ) ${ }^{23}$ or the Malnutrition Universal Screening Tool (MUST). ${ }^{24}$ For patients with poor nutritional status or a high risk of malnutrition, measures should be taken to improve the nutritional status. ${ }^{2,25,26}$ These measures usually consist of dietetic consultation to promote a personalized diet high in energy and proteins, supplemented by the use of oral nutritional supplements or tube feeding when the intake remains insufficient. ${ }^{2,11}$

Information on the prevalence of cachexia and implementation of dietetic interventions in clinical practice could provide relevant insight into the need for potential improvements in nutritional care in current practice. Thus far, these data in esophagogastric cancer are scarce. Moreover, the prognostic effect of cachexia on overall survival (OS) in patients with esophagogastric cancer receiving different treatment modalities has not been explored using real-world data. The aim of this study was to explore the prevalence of pretreatment cachexia, association of pretreatment cachexia with OS, and use of dietetic interventions in patients with esophagogastric cancer using patient-reported outcome measures (PROMs) in a realworld setting (ie, a large national patient cohort).

\section{Materials and Methods}

\section{Data Collection}

Patients with an adenocarcinoma or squamous cell carcinoma of the esophagus, gastroesophageal junction, or stomach diagnosed in 2015 through 2018 in the Netherlands were asked to participate in the Prospective Observational Cohort Study of Esophageal-Gastric Cancer Patients (POCOP), for which PROMs are prospectively collected after informed consent is provided by the patient. ${ }^{27}$ Data on patient and tumor characteristics, treatment, and survival were extracted from the Netherlands Cancer Registry (NCR), which is a population-based registry that covers the total Dutch population of $>17$ million people, for which data are extracted from the hospital's electronic health record system or medical records by trained data managers. Data on vital status were obtained through annual linkage to the Dutch Personal Records Database and were updated until February 1, 2020.

\section{Questionnaires}

Patients included in POCOP were asked to complete computer-administered or paper questionnaires at initial diagnosis (baseline), and 3, 6, 9, 12, 18, 24, and 36 months after inclusion. Patients were considered eligible for inclusion if they completed the baseline questionnaire before resection or before or within a week after start of (neoadjuvant) treatment with chemo(radio)therapy. Results from the baseline and 3-month questionnaire were included, because these questionnaires contained the Dutch Healthy Diet Food Frequency Questionnaire (DHD-FFQ). Information on height, weight, weight loss, and whether patients received dietetic consultation, tube feeding, or oral nutritional supplements was collected in a general questionnaire. Patients who consumed food orally were requested to complete the DHD-FFQ, a validated questionnaire based on Dutch national dietary guidelines including questions about portion sizes of bread, dairy, meat, fish, vegetables, and alcohol consumption. ${ }^{28,29}$ A protein score was calculated based on this DHD-FFQ, ${ }^{30}$ and varied from 0 to 10 . Among the general healthy population, a protein score of 10 is regarded as sufficient intake, whereas $<10$ indicates insufficient intake of the protein sources included in the questionnaire. ${ }^{31}$ For patients with diseaserelated malnutrition, a protein score $<10$ may indicate the need for nutritional support, given that their protein requirements are higher than those of healthy persons.

\section{Weight Loss Parameters}

BMI $\left(\mathrm{kg} / \mathrm{m}^{2}\right)$ was calculated from reported heights and weights retrieved from the questionnaires. Percentage weight difference was calculated from the reported half-year weight loss in the baseline questionnaire, and from weight differences between 2 questionnaires during follow-up. Cachexia was defined as $>5 \%$ body-weight loss or $>2 \%$ in individuals with a BMI of $<20 \mathrm{~kg} / \mathrm{m}^{2}$ according to international consensus criteria. ${ }^{13}$ The criterion for cachexia that included the presence of sarcopenia was not used because data on skeletal muscle mass and strength were unavailable.

\section{Treatment}

Analyses were stratified according to treatment type: (1) neoadjuvant chemoradiotherapy (nCRT) followed by a surgical resection, (2) neoadjuvant chemotherapy (nCT) followed by a resection ( \pm adjuvant chemotherapy), (3) a resection without neoadjuvant treatment, (4) definitive chemoradiotherapy (dCRT; ie, chemotherapy and concurrent long-term radiotherapy without a resection), (5) palliative chemotherapy, and (6) best supportive care (BSC) without chemotherapy or a resection.

\section{Statistical Analysis}

Baseline characteristics and nutritional parameters are displayed with counts and percentages or medians and interquartile ranges (IQRs) and compared using chisquare, Fisher exact, or Mann-Whitney $U$ tests, whichever was appropriate, in patients with versus without pretreatment cachexia. OS was calculated from the day of 
baseline questionnaire completion until the date of death or last follow-up (February 1, 2020). OS was assessed in all patients using the Kaplan-Meier method and groups were compared using the log-rank test, and results were stratified according to treatment type. A multivariable Cox proportional hazard analysis was used to assess the independent prognostic value of cachexia on OS after adjustment for sex, age, performance status, number of comorbidities, primary tumor location, disease stage, histology, and treatment strategy. $P$ values $<.05$ were considered statistically significant. Analyses were performed using SAS 9.4 (SAS Institute Inc.).

\section{Data Availability}

Data supporting the findings of this study are available from the NCR and POCOP PROMs. Restrictions apply to the availability of these data, which were used under license for this study. ${ }^{32}$

\section{Results}

\section{Patient Characteristics}

The total patient cohort included 406 patients with esophagogastric cancer who completed the baseline questionnaire before start of treatment (Figure 1).
Of these, 244 completed the 3-month follow-up questionnaire. Median time between completion of the baseline and 3-month questionnaire was 92 days (IQR, 88-97 days).

Of all 406 patients, $197(49 \%)$ had pretreatment cachexia (Table 1 ), and $21 \%$ reported that they lost $>10 \%$ of their body weight within 6 months before diagnosis. Compared with patients without cachexia, those with cachexia were more often female, had more frequently a performance status of $\geq 2$, and had a higher disease stage.

\section{Cachexia and OS}

OS was significantly higher in patients without versus with pretreatment cachexia $(P<.01$; Figure 2$)$, with a median survival of 41 months (25th percentile, 14.7 months) versus 19 months (25th percentile, 8.4 months), respectively. When OS was analyzed per treatment type, a numerical difference in survival between patients with and without cachexia was seen in almost all treatment groups, but this difference was not statistically significant (supplemental eFigure 1, available with this article at JNCCN.org).

In multivariable analyses, after adjustment for sex, age, performance status, number of comorbidities, primary tumor location, disease stage, histology,

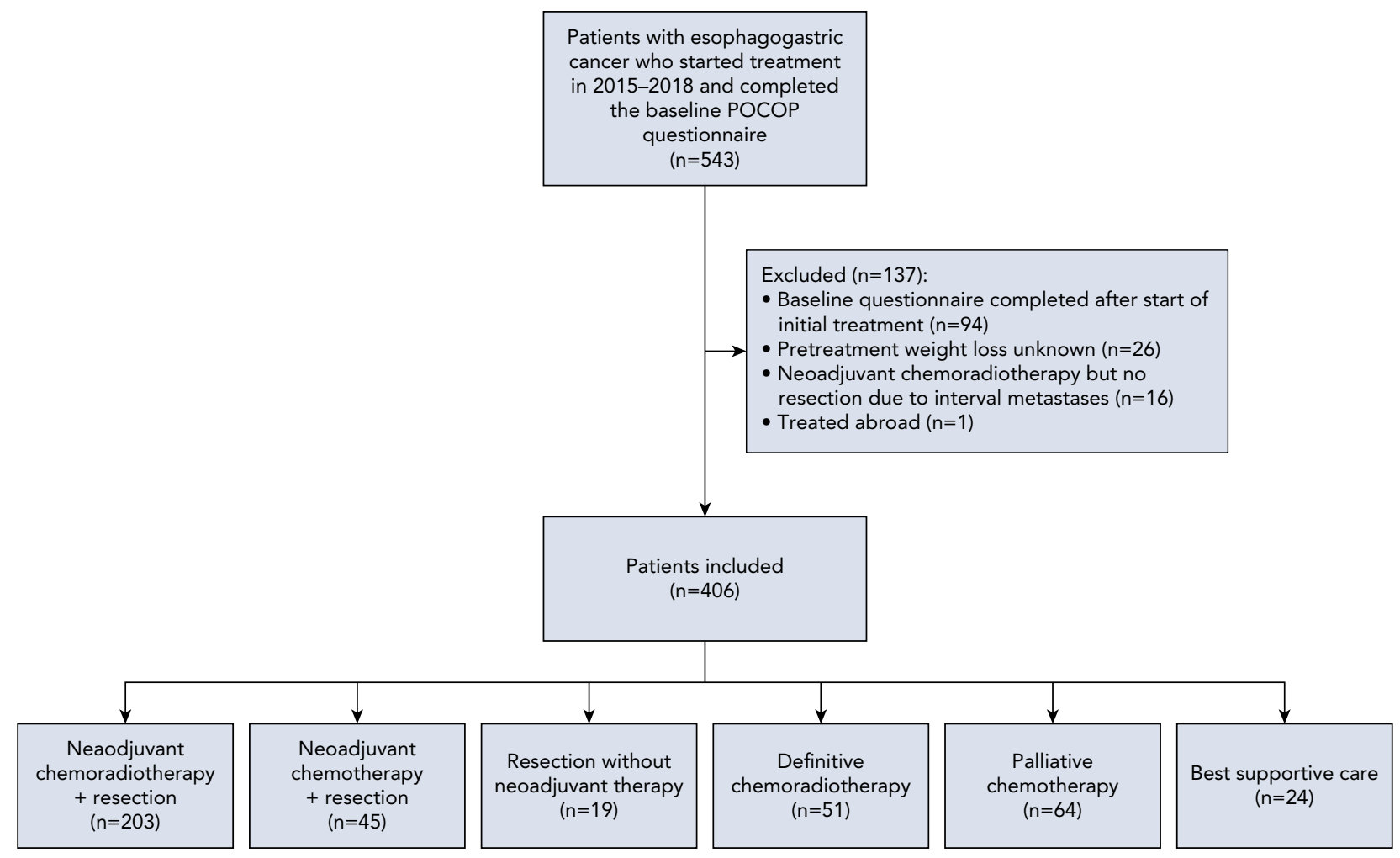

Figure 1. Flow diagram of patient inclusion. All 406 included patients completed the baseline questionnaire; 244 (64\%) of 383 patients were still alive at 3-month follow-up.

Abbreviation: POCOP, Prospective Observational Cohort Study of Esophageal-Gastric Cancer Patients. 
Table 1. Patient Characteristics at Baseline

\begin{tabular}{|c|c|c|c|}
\hline & $\begin{array}{c}\text { Pretreatment } \\
\text { Cachexia } \\
\text { n (\%) }\end{array}$ & $\begin{array}{l}\text { No Pretreatment } \\
\text { Cachexia } \\
\text { n (\%) }\end{array}$ & $\begin{array}{c}P \\
\text { Value }\end{array}$ \\
\hline Total, $\mathrm{n}$ & 197 & 209 & \\
\hline Female & $58(29)$ & $38(18)$ & $.008^{a}$ \\
\hline Age, median (IQR), y & $66(59-71)$ & $66(60-71)$ & $.382^{\mathrm{b}}$ \\
\hline$<55 \mathrm{y}$ & $24(12)$ & $20(10)$ & $.809^{a}$ \\
\hline $55-64$ y & $62(31)$ & $63(30)$ & \\
\hline $65-74 y$ & $82(42)$ & $93(44)$ & \\
\hline$\geq 75$ y & $29(15)$ & $33(16)$ & \\
\hline Performance status & & & $.010^{a}$ \\
\hline 0 or 1 & $141(72)$ & $162(78)$ & \\
\hline$\geq 2$ & $15(8)$ & $3(1)$ & \\
\hline Unknown & $41(21)$ & $44(21)$ & \\
\hline Comorbidities & & & $.707^{\mathrm{a}}$ \\
\hline 0 & $60(30)$ & $62(30)$ & \\
\hline 1 & $49(25)$ & $48(23)$ & \\
\hline$\geq 2$ & $56(28)$ & $70(33)$ & \\
\hline Unknown & $32(16)$ & $29(14)$ & \\
\hline Tumor location & & & $.341^{\mathrm{a}}$ \\
\hline Esophagus & $149(76)$ & $156(75)$ & \\
\hline GEJ or cardia & $25(13)$ & $20(10)$ & \\
\hline Stomach & $23(12)$ & $33(16)$ & \\
\hline Histology & & & $.464^{c}$ \\
\hline Adenocarcinoma & $147(75)$ & $167(80)$ & \\
\hline $\begin{array}{l}\text { Squamous cell } \\
\text { carcinoma }\end{array}$ & $46(23)$ & $38(18)$ & \\
\hline Carcinoma NOS & $4(2)$ & $4(2)$ & \\
\hline Disease stage ${ }^{d}$ & & & $.028^{a}$ \\
\hline I & $28(14)$ & $56(27)$ & \\
\hline$\|$ & $22(11)$ & $18(9)$ & \\
\hline III & $89(45)$ & $83(40)$ & \\
\hline IV & $47(24)$ & $38(18)$ & \\
\hline$x$ & $11(6)$ & $14(7)$ & \\
\hline Reported BMI, kg/m² & & & $<.001^{a}$ \\
\hline$<18.5$ & $12(6)$ & $1(0)$ & \\
\hline $18.5-25$ & $109(55)$ & $80(38)$ & \\
\hline$>25$ & 76 (39) & $128(61)$ & \\
\hline
\end{tabular}

(continued)

and treatment strategy, cachexia was independently associated with OS (adjusted hazard ratio [HR], 1.37, 95\% CI, 1.03-1.83; Table 2).

\section{Nutritional Screening and Dietetic Consultation}

A total of $65 \%$ of patients with cachexia received dietetic consultation before start of treatment compared with $27 \%$ of those without cachexia. The proportion of patients

\begin{tabular}{|c|c|c|c|}
\hline & $\begin{array}{c}\text { Pretreatment } \\
\text { Cachexia } \\
\text { n (\%) }\end{array}$ & $\begin{array}{l}\text { No Pretreatment } \\
\text { Cachexia } \\
\text { n (\%) }\end{array}$ & $\begin{array}{c}P \\
\text { Value }\end{array}$ \\
\hline \multicolumn{3}{|c|}{ Reported half-year weight loss } & $<.001^{\mathrm{a}}$ \\
\hline $\begin{array}{l}\text { Stable weight }(<2 \% \\
\text { weight loss) }\end{array}$ & $0(0)$ & $146(70)$ & \\
\hline $\begin{array}{l}\text { Medium weight loss } \\
(2 \%-10 \%)\end{array}$ & $112(57)$ & $63(30)$ & \\
\hline $\begin{array}{l}\text { Severe weight loss } \\
(>10 \%)\end{array}$ & $85(43)$ & $0(0)$ & \\
\hline
\end{tabular}

Baseline characteristics of included patients stratified for pretreatment cachexia (ie, reported half-year body weight loss of $>5 \%$ or $>2 \%$ in patients with a reported BMI of $<20 \mathrm{~kg} / \mathrm{m}^{2}$ ).

Abbreviations: BMI, body mass index; GEJ, gastroesophageal junction; IQR interquartile range; NOS, not otherwise specified.

aChi-square test.

bMann-Whitney $U$ test.

cFisher exact test.

dDisease stage according to 7th edition of the AJCC Cancer Staging Manual.

with pretreatment cachexia was highest in those who received BSC $(67 \%)$, followed by those treated with palliative chemotherapy (59\%), nCRT + resection $(47 \%)$, dCRT $(43 \%)$, nCT + resection $(42 \%)$, and a resection without neoadjuvant therapy (32\%) (Figure 3). A total of $71 \%$ of patients with pretreatment cachexia treated with nCRT + resection or dCRT received dietetic consultation before start of treatment compared with $68 \%$ of those treated with palliative chemotherapy, $42 \%$ of those treated with nCT + resection, and 33\% who underwent a resection only. More than half of the patients treated with nCRT + resection, dCRT, palliative chemotherapy, or BSC used oral nutritional supplements (Figure 3).

Overall, the protein score was unknown in 78 patients, which includes those who received tube feeding $(n=28)$ and other patients who did not complete the DHD-FFQ. Of the 328 remaining patients, $267(81 \%)$ had a protein score of $<10$. Patients with a protein score of 10 received oral nutritional supplements half as often as those with a protein score $<10$ ( $16 \%$ vs $33 \%$, respectively; $P=.009$ ), whereas no difference was seen in the proportion of patients who were referred for dietetic support (34\% vs $35 \%$, respectively; $P=.952)$. A total of $152(78 \%)$ of the 194 patients who completed the DHD-FFQ at 3 months had a protein score of $<10$. The proportion of patients with cachexia who had a protein score of $<10$ is shown in Figure 3.

Of 103 patients with pretreatment cachexia who completed the baseline and 3-month questionnaires, $60(58 \%)$ received dietetic consultation at baseline. Median half-year pretreatment weight difference was $-9 \%$ (IQR, $-13 \%$ to $-7 \% \%$ ) in the patients with cachexia who received dietetic consultation at baseline, and $-8 \%$ (IQR, $-11 \%$ to $-7 \%$ ) in the 43 patients with cachexia who did not $(P=.222)$. Weight of the patients who received dietetic consultation was stable in the 3 months thereafter 


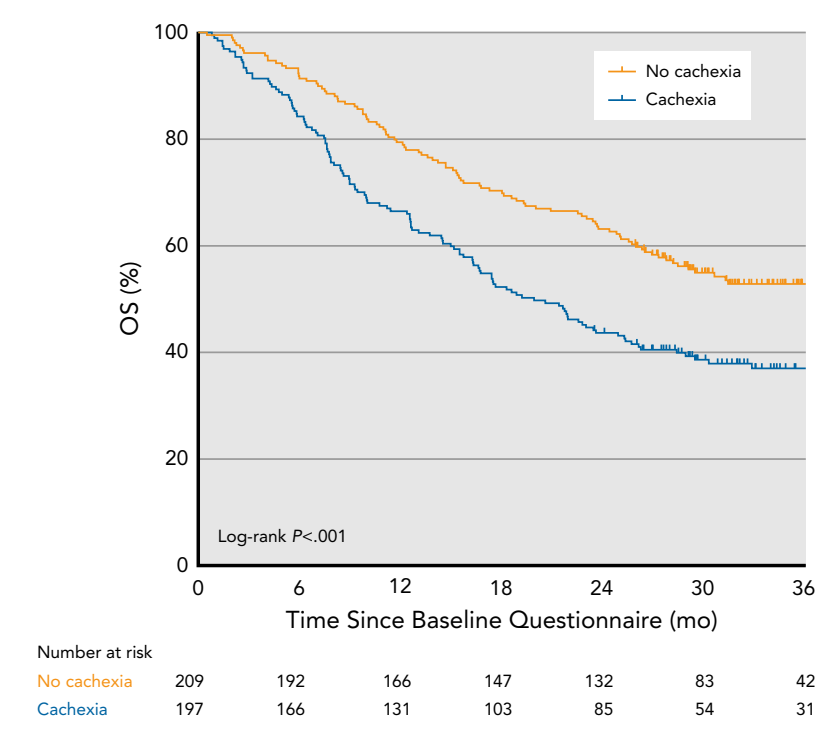

Figure 2. Overall survival (OS) in all patients stratified for pretreatment cachexia after completion of the baseline questionnaire and those who completed at least the baseline questionnaire.

(median weight difference, $0 \%$ [IQR, $-3 \%$ to $2 \%]$ ), and differed statistically significantly from patients who were not referred at baseline (median weight difference, $-2 \%$ [IQR, $-6 \%$ to $2 \%$ ]; $P=.047$ ).

\section{Discussion}

Pretreatment cachexia was observed in $48 \%$ of 406 patients with esophagogastric cancer and associated with decreased survival. The fact that more than one-third of patients with cachexia did not receive dietetic consultation before start of treatment is worrisome, because body weight loss at 3 months was lower in patients who were referred to a dietician compared with those who were not referred at baseline. Our findings suggest an urgent need for awareness of the importance of nutritional screening and subsequent referral for dietetic interventions at an early stage in multimodal treatment of esophagogastric cancer.

Preventing cachexia is important, because it can ultimately result in a nonreversible, refractory state that is associated with impaired response to anticancer treatment and limited life expectancy. ${ }^{12}$ A previous study of patients with a low initial BMI showed that even subtle weight loss can result in decreased survival, ${ }^{33}$ independent of performance status and disease stage. The presence of pretreatment cachexia, which was defined as self-reported half-year body-weight loss of $>5 \%$ or of $>2 \%$ in patients with a BMI $<20 \mathrm{~kg} / \mathrm{m}^{2}$ according to internationally accepted criteria, ${ }^{13}$ was independently associated with shorter OS in our study, emphasizing the need for early screening and interventions and underlining the importance of awareness of the presence of cachexia among physicians to prevent (further) weight loss in every patient with esophagogastric cancer.

Unfortunately, information on depletion of skeletal muscle mass and strength was not available, which possibly resulted in an underestimation of patients with cachexia because muscle mass and strength are part of one of the criteria for the determination of cancer cachexia. ${ }^{13}$ Nevertheless, nearly one-half of the patients already had cachexia according to the criteria concerning weight loss and BMI only..$^{13}$ Ideally, assessment of weight loss, BMI, and skeletal muscle mass will be routinely performed in the future to identify all patients with cachexia. ${ }^{1}$

Nutritional interventions can help stop weight loss and prevent (evolvement of) cachexia. ${ }^{2,9,25,26,34}$ We found that patients with cachexia who were referred to a dietician before start of treatment had less weight loss in the subsequent 3 months compared with patients who were not referred before start of treatment. Unfortunately, we could not evaluate the effect of dietetic support on clinical outcomes (eg, quality of life and survival) in this retrospective study. Nevertheless, we did observe that more than one-third of the patients with pretreatment cachexia were not referred to a dietician. Possible causes of nonreferral could include lack of awareness to the presence of cachexia, and inadequate or absence of nutritional screening, which could result in insufficient application of nutritional interventions. ${ }^{2}$ Moreover, because cancer-related malnutrition can develop at any time, including during treatment, ${ }^{35}$ nutritional risk screening should be performed on a regular basis. It is therefore recommended that each hospital involved in the treatment of cancer should incorporate standard procedures for early and routine screening for malnutrition and implement nutritional interventions for patients in both in-hospital and out-ofhospital settings. ${ }^{2}$ This approach should ideally be multidisciplinary, in which the primary responsible physician takes care of the referral, and works closely with the dietician while staying involved in the nutritional status and interventions. These interventions usually include a protein- and energy-enriched diet, possibly supplemented by oral nutritional supplements or tube feeding, adapted to the specific needs of the patient. ${ }^{2,11}$

Use of conventional screening tools for nutritional risk assessment that are validated in hospitalized patients with cancer (ie, $\mathrm{SNAQ}^{23}$ and $\mathrm{MUST}^{24}$ ) could underestimate malnutrition in the outpatient clinic. ${ }^{36,37}$ Retrospectively completing these nutritional screening tools using data from the present study showed that SNAQ and MUST only identified three-quarters and one-half of patients who already had cachexia as being at high risk of malnutrition, respectively. The reason for this is that in patients 
Table 2. Cox Regression Analyses for Overall Survival

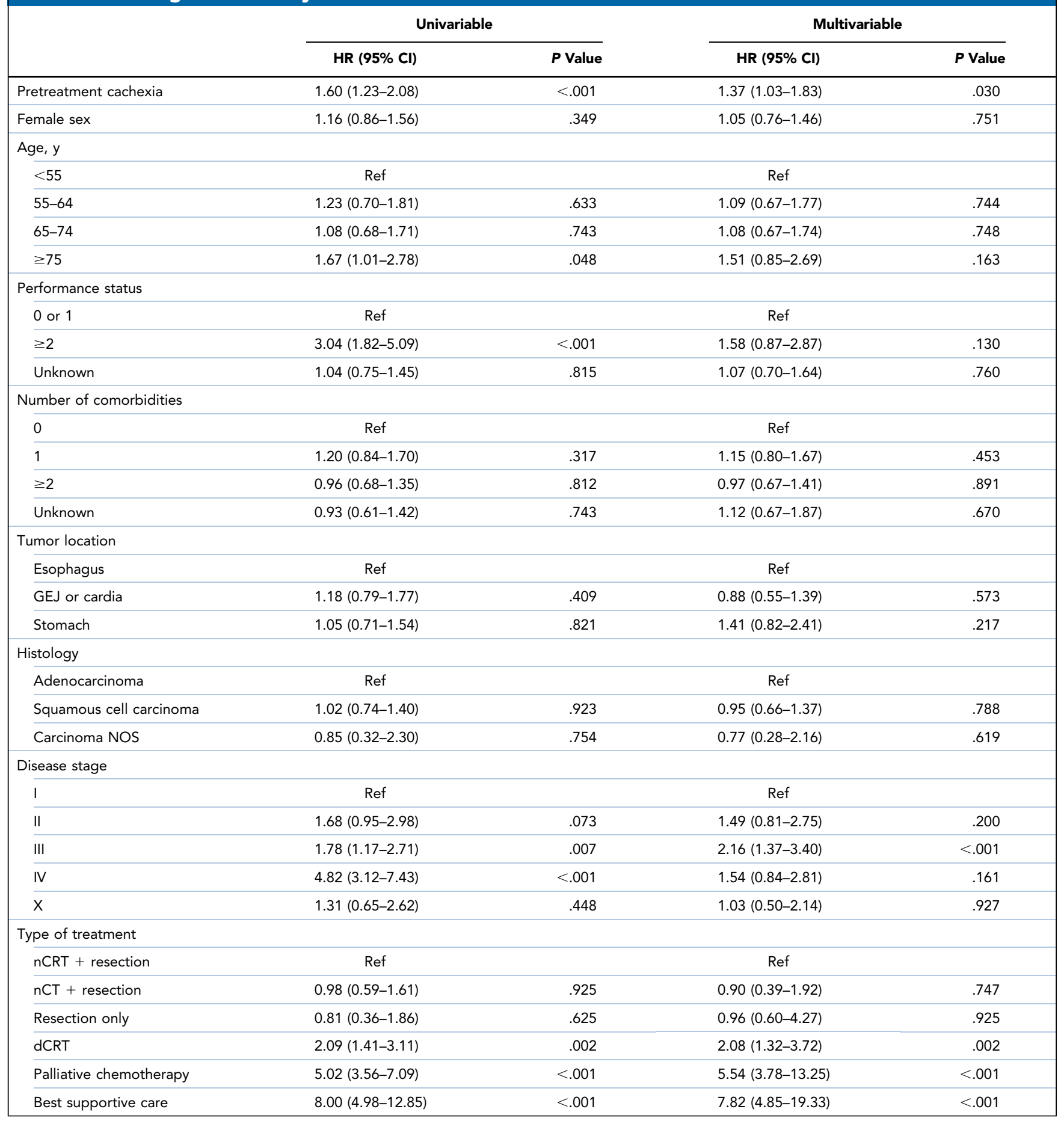

Abbreviations: dCRT, definitive chemoradiotherapy; GEJ, gastroesophageal junction; HR, hazard ratio; $\mathrm{nCRT}$, neoadjuvant chemoradiotherapy; $\mathrm{nCT}$, neoadjuvant chemotherapy; NOS, not otherwise specified.

with a low BMI, a lower percentage of body weight loss is already defined as cachexia. ${ }^{13,23}$ An option for screening is to ascertain the presence of cachexia using just the definition that includes body weight loss and BMI, or to use screening tools that include both parameters because they take into consideration the vulnerability of patients with a low BMI, ${ }^{33}$ such as the Patient-Generated Subjective Global Assessment Short-Form. ${ }^{38}$ 


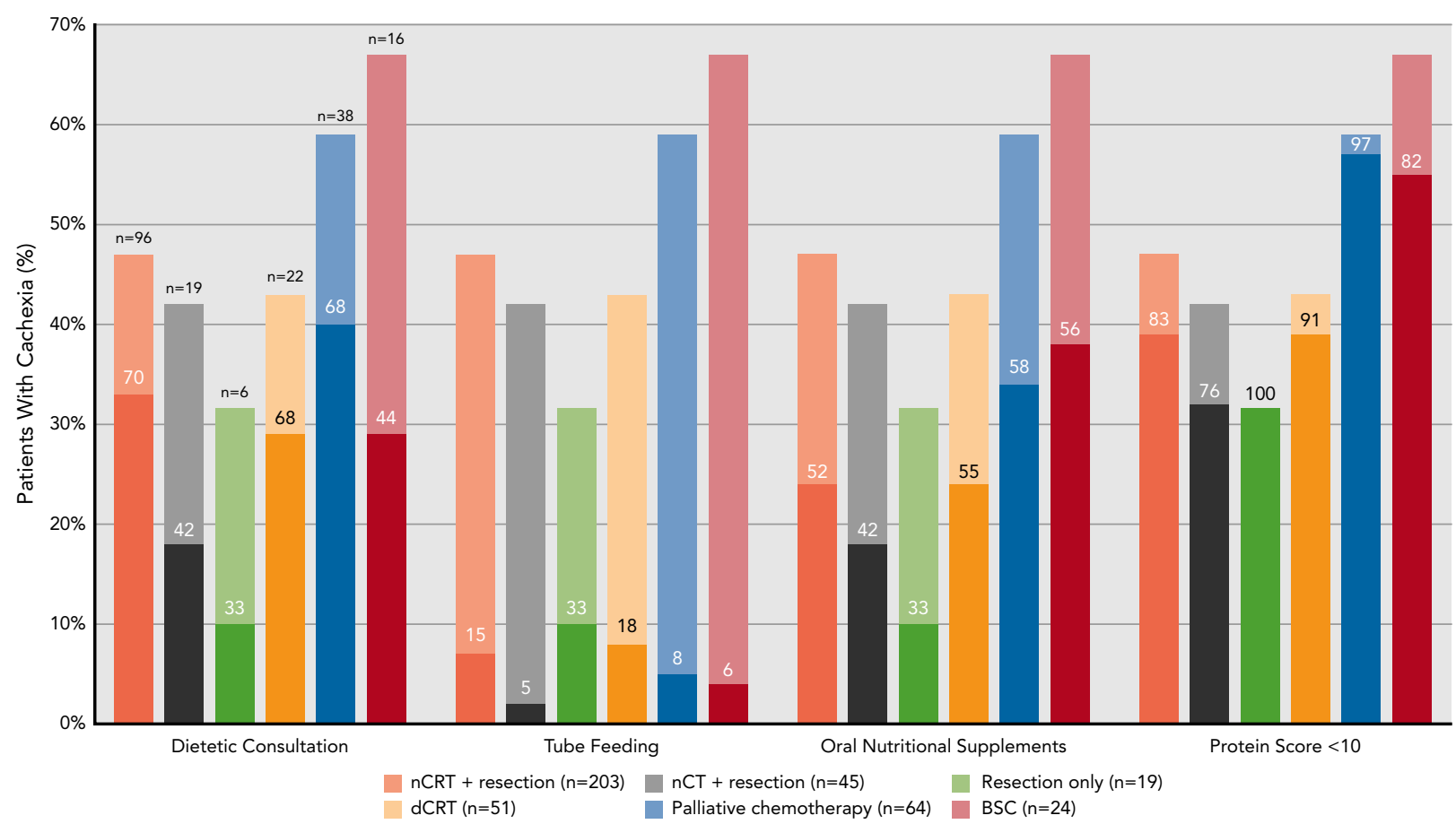

Figure 3. Dietetic interventions, use of nutritional supplements and protein score in patients with cachexia stratified per type of treatment at baseline. The percentages within the bars reflect the proportion of patients with pretreatment cachexia. Protein scores of patients with cachexia who completed the DHD-FFQ are shown $(n=135)$.

Abbreviations: BSC, best supportive care; DHD-FFQ, the Dutch Healthy Diet Food Frequency Questionnaire; dCRT, definitive chemoradiotherapy; nCRT, neoadjuvant chemoradiotherapy; $\mathrm{nCT}$, neoadjuvant chemotherapy; SNAQ, Short Nutritional Assessment Questionnaire.

Causes of cancer-related malnutrition and cachexia are multifactorial and include reduced food and/or protein intake, and metabolic changes due to tumorand treatment-related factors. ${ }^{10,19}$ We also found inadequate food intake in a large part of our population. A protein score of $<10$ was found in $81 \%$ of the patients who completed the DHD-FFQ. Among the general healthy population, this score indicates insufficient intake of the protein sources included in the DHDFFQ. Moreover, in patients with cancer, a higher protein intake $(1-1.5 \mathrm{~g} / \mathrm{kg} / \mathrm{d})$ is recommended than in healthy persons, ${ }^{2}$ suggesting that the number of patients with inadequate protein intake from these sources is even higher. Furthermore, only $33 \%$ of the patients with a protein score of $<10$ received oral nutritional supplements. Both findings may have contributed to the reported weight loss.

Although dietetic consultation can be helpful in preventing weight loss, it should be noted that cachexia is not completely reversible by nutritional therapy alone..$^{10,12}$ Treatment and prevention of malnutrition and cachexia should therefore occur early in the cancer treatment trajectory and multimodal, and at least consist of physical therapy in addition to dietetic consultation and provision of energy- and protein-enriched dietary advice, possibly supplemented with oral nutritional supplements or (par)enteral nutrition. ${ }^{2}$

A strength of this study is that real-world data were included, which provide a good indication of current practice. However, there were also some limitations. One of the limitations is that there is probably a selection bias of patients who were included in POCOP, with the result that the study population may not reflect the actual patient population and patients with a higher tumor stage or worse nutritional status may not be represented adequately. Because some results were based on patient-reported outcomes, misinterpretations due to inadequate or incomplete reporting cannot be excluded. Unfortunately, we did not know if patients were formally screened for the presence of malnutrition. Moreover, we could not calculate exact protein intake, because the protein score only includes protein sources mostly contributing to intake in a healthy population, and does not include supplements. Furthermore, it would have been interesting to compare treatment intensity and tolerability between patients with and without cachexia, but this was not possible because of the limited number of patients within the treatment groups and missing data. The small sample sizes within the treatment groups also hindered us from 
stratifying for treatment strategy in the Cox proportional hazard analyses. Because univariable HRs on OS cachexia showed the same pattern in all treatment groups, we put all patients in one model and adjusted for treatment strategy. Lastly, weight loss comparisons were only performed in patients who completed the baseline and 3-month questionnaire, which could have resulted in a selection bias of patients who survived a longer period and were probably in a better condition.

\section{Conclusions}

Cachexia is common in esophagogastric cancer, even before start of treatment, and associated with decreased survival. Physicians should therefore be aware of the risk, presence, and consequences of cachexia already present at initial diagnosis. In the multimodal treatment trajectory, early and adequate nutritional screening and referral for dietetic support are of major importance to prevent weight loss and improve survival outcomes, and are indicated for every patient with esophagogastric cancer in both the in-hospital and out-of-hospital settings.

\section{Acknowledgments}

The authors thank the registration team of the Netherlands Comprehensive Cancer Organisation (IKNL) for the collection of data for the Netherlands Cancer Registry, and the POCOP team for collecting the questionnaires.

Submitted April 19, 2020; accepted for publication July 1, 2020. Published online January 8, 2021.

Author contributions: Study concept and design: Dijksterhuis, Latenstein, van Oijen, Beijer, van Laarhoven. Data acquisition: Dijksterhuis, van Kleef. Data analysis: Dijksterhuis. Manuscript preparation: Dijksterhuis, Latenstein, van Oijen, Beijer, van Laarhoven. Final approval of manuscript: All authors.

Disclosures: Dr. Verhoeven has disclosed that he has received grant/research support from Bristol-Myers Squibb and Roche. Dr. van Oijen has disclosed that he has received grant/research support from Bristol-Myers Squibb, Merck Serono, Nordic, Roche, and Servier. Dr. van Laarhoven has disclosed that he has received consulting fees from Bristol-Myers Squibb, Celgene, Lilly, Nordic Pharma, and Servier, and grant/research support from Bayer, Bristol-Myers Squibb, Celgene, Lilly, Merck Serono, Merck Sharpe \& Dohme, Nordic, Philips, Roche, and Servier. The remaining authors have disclosed that they have not received any financial consideration from any person or organization to support the preparation, analysis, results, or discussion of this article.

Funding: This work was supported by funding from the Dutch Cancer Society.

Disclaimer: According to the Central Committee on Research Involving Human Subjects, this type of study does not require approval from an ethics committee in the Netherlands. The study was approved by the Privacy Review Board of the Netherlands Cancer Registry and the scientific committee of the Dutch Upper GI Cancer Group. Patients who participated in the Prospective Observational Cohort Study of Oesophageal-Gastric Cancer Patients (POCOP) provided written informed consent.

Correspondence: Hanneke W.M. van Laarhoven, MD, Department of Medical Oncology, Amsterdam UMC, University of Amsterdam, Cancer Center Amsterdam, Meibergdreef 9, D3-221.1, 1105 AZ Amsterdam, the

Netherlands. Email: h.vanlaarhoven@amsterdamumc.nl

\section{References}

1. Cederholm T, Jensen GL, Correia MITD et al. GLIM criteria for the diagnosis of malnutrition-a consensus report from the global clinical nutrition community. J Cachexia Sarcopenia Muscle 2019;10:207-217.

2. Arends J, Bachmann P, Baracos $V$, et al. ESPEN guidelines on nutrition in cancer patients. Clin Nutr 2017;36:11-48

3. Mak M, Bell K, Ng W, et al. Nutritional status, management and clinical outcomes in patients with esophageal and gastro-oesophageal cancers: a descriptive study. Nutr Diet 2017;74:229-235.

4. Miller KR, Bozeman MC. Nutrition therapy issues in esophageal cancer. Curr Gastroenterol Rep 2012;14:356-366.

5. Rosania R, Chiapponi C, Malfertheiner $P$, et al. Nutrition in patients with gastric cancer: an update. Gastrointest Tumors 2016;2:178-187.

6. Marshall KM, Loeliger J, Nolte L, et al. Prevalence of malnutrition and impact on clinical outcomes in cancer services: a comparison of two time points. Clin Nutr 2019;38:644-651.

7. de Vries YC, Helmich E, Karsten MDA, et al. The impact of chemosensory and food-related changes in patients with advanced oesophagogastric cancer treated with capecitabine and oxaliplatin: a qualitative study. Support Care Cancer 2016;24:3119-3126.

8. Van Cutsem E, Arends J. The causes and consequences of cancerassociated malnutrition. Eur J Oncol Nurs 2005;9(Suppl 2):S51-63.

9. Ravasco P, Monteiro-Grillo I, Marques Vidal P, et al. Impact of nutrition on outcome: a prospective randomized controlled trial in patients with head and neck cancer undergoing radiotherapy. Head Neck 2005;27: 659-668.

10. Baracos VE. Cancer-associated malnutrition. Eur J Clin Nutr 2018;72 1255-1259.

11. de van der Schueren MAE. Use and effects of oral nutritional supplements in patients with cancer. Nutrition 2019;67-68:110550.

12. Anandavadivelan $P$, Lagergren P. Cachexia in patients with oesophageal cancer. Nat Rev Clin Oncol 2016;13:185-198.

13. Fearon K, Strasser F, Anker SD, et al. Definition and classification of cancer cachexia: an international consensus. Lancet Oncol 2011;12:489-495.

14. Baracos VE, Martin L, Korc M, et al. Cancer-associated cachexia. Nat Rev Dis Primers 2018:4:17105.
15. Järvinen $T$, llonen I, Kauppi J, et al. Loss of skeletal muscle mass during neoadjuvant treatments correlates with worse prognosis in esophageal cancer: a retrospective cohort study. World J Surg Oncol 2018;16:27.

16. Elliott JA, Doyle SL, Murphy CF, et al. Sarcopenia: prevalence, and impact on operative and oncologic outcomes in the multimodal management of locally advanced esophageal cancer. Ann Surg 2017;266:822-830.

17. Andreyev HJ, Norman AR, Oates J, et al. Why do patients with weight loss have a worse outcome when undergoing chemotherapy for gastrointestinal malignancies? Eur J Cancer 1998;34:503-509.

18. Capuano G, Grosso A, Gentile P, et al. Adult height and head and neck cancer: a pooled analysis within the INHANCE Consortium. Head Neck 2008;30:503-508.

19. Martin L, Kubrak C. How much does reduced food intake contribute to cancer-associated weight loss? Curr Opin Support Palliat Care 2018;12: 410-419.

20. Dijksterhuis WPM, Pruijt MJ, van der Woude SO, et al. Association between body composition, survival, and toxicity in advanced esophagogastric cancer patients receiving palliative chemotherapy. J Cachexia Sarcopenia Muscle 2019;10:199-206.

21. Lakenman P, Ottens-Oussoren K, Witvliet-van Nierop J, et al. Handgrip strength is associated with treatment modifications during neoadjuvant chemoradiation in patients with esophageal cancer. Nutr Clin Pract 2017 32:652-657

22. Ligthart-Melis GC, Weijs PJM, te Boveldt ND, et al. Dietician-delivered intensive nutritional support is associated with a decrease in severe postoperative complications after surgery in patients with esophageal cancer. Dis Esophagus 2013;26:587-593.

23. Kruizenga HM, Seidell JC, de Vet HCW, et al. Development and validation of a hospital screening tool for malnutrition: the Short Nutritional Assessment Questionnaire (SNAQ). Clin Nutr 2005;24:75-82.

24. Boléo-Tomé $\mathrm{C}$, Monteiro-Grillo I, Camilo $\mathrm{M}$, et al. Validation of the Malnutrition Universal Screening Tool (MUST) in cancer. Br J Nutr 2012; 108:343-348.

25. de van der Schueren MAE, Laviano A, Blanchard $H$, et al. Systematic review and meta-analysis of the evidence for oral nutritional intervention 
on nutritional and clinical outcomes during chemo(radio)therapy: current evidence and guidance for design of future trials. Ann Oncol 2018;29: 1141-1153.

26. Bozzetti F. Nutritional support in patients with oesophageal cancer Support Care Cancer 2010;18(Suppl 2):S41-50.

27. Coebergh van den Braak RRJ, van Rijssen LB, van Kleef JJ, et al. Nationwide comprehensive gastro-intestinal cancer cohorts: the 3P initiative. Acta Oncol 2018;57:195-202.

28. van Lee L, Feskens EJM, Meijboom S, et al. Evaluation of a screener to assess diet quality in the Netherlands. Br J Nutr 2016;115: 517-526.

29. Health Council of the Netherlands. Guidelines for a Healthy Diet 2006 The Hague, Netherlands: Health Council of the Netherlands; 2006. Publication no. 2006/21E.

30. van Doorn-van Atten MN, Haveman-Nies A, van Bakel MM, et al. Effects of a multi-component nutritional telemonitoring intervention on nutritional status, diet quality, physical functioning and quality of life of community-dwelling older adults. Br J Nutr 2018;119. 1185-1194.

31. Gezondheidsraad [Health Council of the Netherlands]. Richtlijnen goede voiding 2015 [Guidelines for a healthy diet 2015]. Accessed October 30, 2020. Available at: https://www.gezondheidsraad.nl/ documenten/adviezen/2015/11/04/richtlijnen-goede-voeding2015
32. DUCG. Dutch Upper GI Cancer Group. DUCG 2015. Available at: www. ducg.nl

33. Martin L, Senesse P, Gioulbasanis I, et al. Diagnostic criteria for the classification of cancer-associated weight loss. J Clin Oncol 2015;33: 90-99.

34. Ravasco P, Monteiro-Grillo I, Vidal PM, et al. Dietary counseling improves patient outcomes: a prospective, randomized, controlled trial in colorecta cancer patients undergoing radiotherapy. J Clin Oncol 2005;23: 1431-1438.

35. Rietveld SCM, Witvliet-van Nierop JE, Ottens-Oussoren K, et al. The prediction of deterioration of nutritional status during chemoradiation therapy in patients with esophageal cancer. Nutr Cancer 2018;70: 229-235.

36. Helfenstein SF, Uster A, Rühlin M, et al. Are four simple questions able to predict weight loss in outpatients with metastatic cancer? A prospective cohort study assessing the Simplified Nutritional Appetite Questionnaire. Nutr Cancer 2016;68:743-749.

37. Leistra $\mathrm{E}$, Langius JAE, Evers $\mathrm{AM}$, et al. Validity of nutritional screening with MUST and SNAQ in hospital outpatients. Eur J Clin Nutr 2013;67: 738-742.

38. Abbott J, Teleni L, McKavanagh D, et al. Patient-Generated Subjective Global Assessment Short Form (PG-SGA SF) is a valid screening tool in chemotherapy outpatients. Support Care Cancer 2016, 24:3883-3887.

See JNCCN.org for supplemental online content.

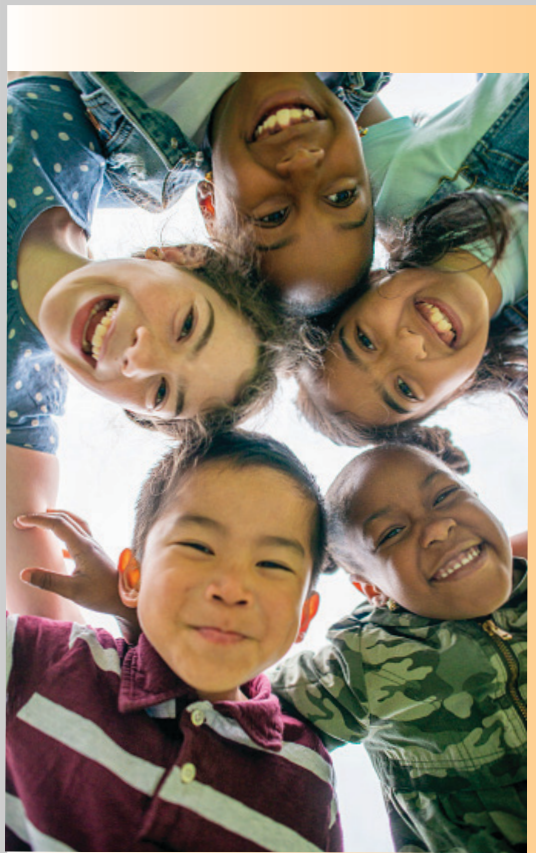

This activity is supported by educational funding provided by Amgen. This activity is supported by educational grants from Novartis and Seagen Inc. (formerly Seattle Genetics, Inc.).
National Comprehensive
NCCN
Cancer Network

\section{New NCCN Guidelines for Pediatric Cancers:}

\section{A Webinar Series}

Multidisciplinary Management of Wilms Tumor (Nephroblastoma)

Friday, February 19, 2021 | 10:30 - 11:30 AM EST

Frank M. Balis, MD

The Children's Hospital of Philadelphia/

Abramson Cancer Center at the University of Pennsylvania

Christine E. Hill-Kayser, MD

University of Pennsylvania

Abramson Cancer Center at the University of Pennsylvania

Peter Mattei, MD

The Children's Hospital of Philadelphial

Abramson Cancer Center at the University of Pennsylvania

Visit education.nccn.org/pediatric-cancers to register for upcoming webinars. 
Supplemental online content for:

\section{Cachexia and Dietetic Interventions in Patients With Esophagogastric Cancer: A Multicenter Cohort Study}

Willemieke P.M. Dijksterhuis, MD; Anouk E.J. Latenstein, MD; Jessy Joy van Kleef, MSc; Rob H.A. Verhoeven, PhD; Jeanne H.M. de Vries, PhD; Marije Slingerland, MD, PhD; Elles Steenhagen, BSc; Joos Heisterkamp, MD, PhD; Liesbeth M. Timmermans, PhD; Marian A.E. de van der Schueren, PhD; Martijn G.H. van Oijen, PhD;

Sandra Beijer, PhD; and Hanneke W.M. van Laarhoven, MD, PhD

J Natl Compr Canc Netw 2021;19(2):144-152

eFigure 1: Overall Survival of Patients Stratified for Pretreatment Cachexia by Treatment Type 
A

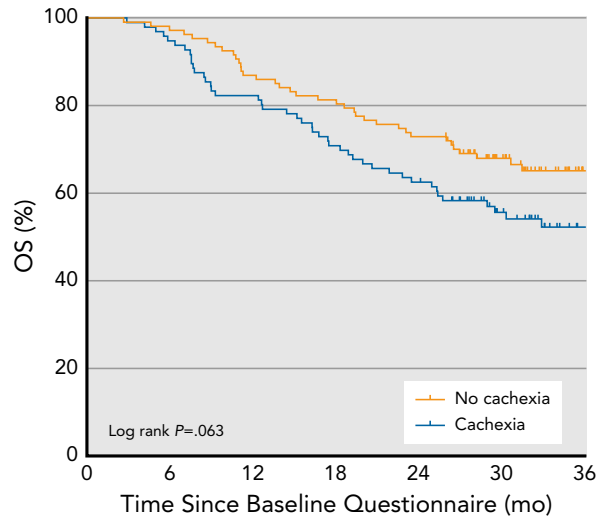

Number at risk

$\begin{array}{lrrrrrrr}\text { No cachexia } & 107 & 104 & 93 & 87 & 78 & 52 & 24 \\ \text { Cachexia } & 96 & 91 & 79 & 68 & 60 & 38 & 20\end{array}$

\section{C}

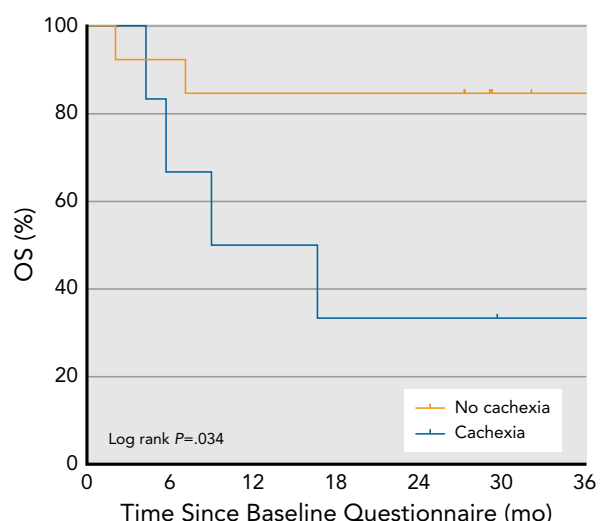

Number at risk

$\begin{array}{lrrrrrr}\text { No cachexia } & 13 & 12 & 11 & 11 & 11 & 6 \\ \text { Cachexia } & 6 & 4 & 3 & 2 & 2 & 1\end{array}$

\section{E}

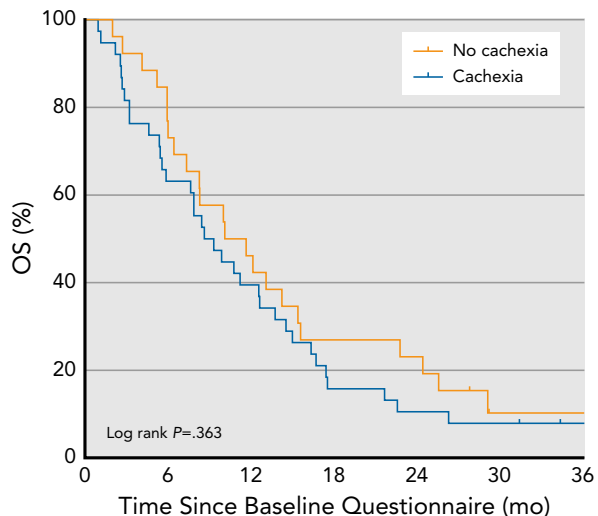

Number at risk

$\begin{array}{llllllll}\text { No cachexia } & 26 & 20 & 12 & 7 & 6 & 1 & 1\end{array}$
B

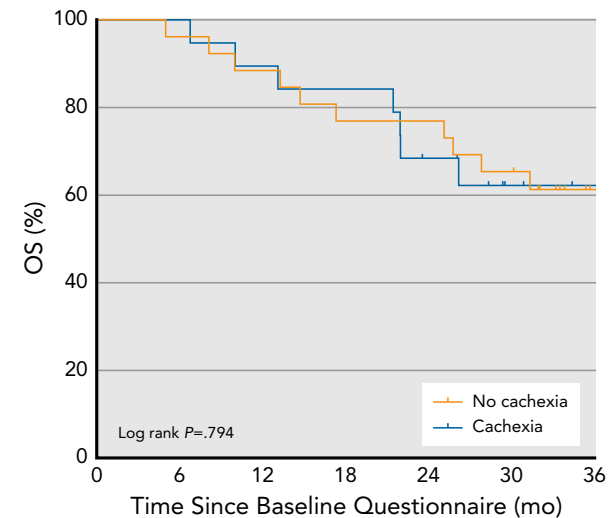

Number at risk

$\begin{array}{lrrrrrrr}\text { No cachexia } & 26 & 25 & 23 & 20 & 20 & 17 & 8 \\ \text { Cachexia } & 19 & 19 & 17 & 17 & 12 & 7 & 5\end{array}$

D

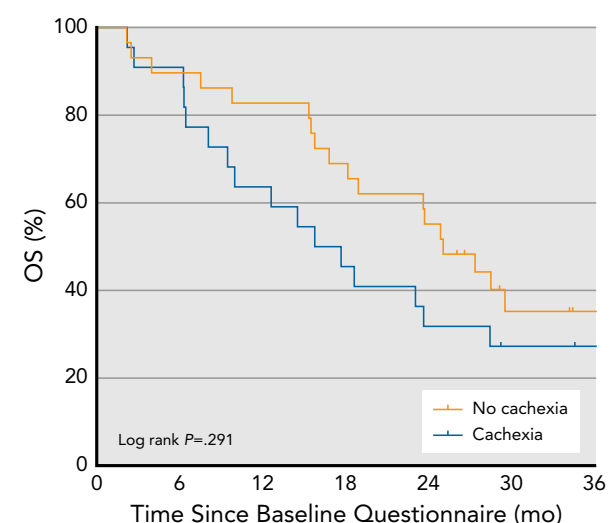

Number at risk

$\begin{array}{llllllll}\text { No cachexia } & 29 & 26 & 24 & 20 & 16 & 7 & 5\end{array}$

F

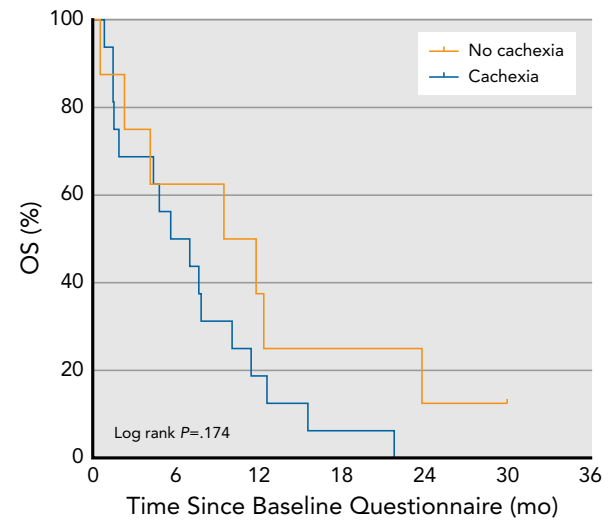

$\begin{array}{lrrrrrrr}\text { No cachexia } & 8 & 5 & 3 & 3 & 1 & 0 & 0 \\ \text { Cachexia } & 16 & 8 & 3 & 1 & 0 & 0 & 0\end{array}$

eFigure 1. Overall survival (OS) of patients stratified for pretreatment cachexia by treatment type: (A) neoadjuvant chemoradiotherapy resection, (B) neoadjuvant chemotherapy + resection, (C) resection only, (D) definitive chemoradiotherapy, (E) palliative chemotherapy, and (F) best supportive care. 